\title{
Potencial melífero de dos apiarios ubicados en los cantones Cevallos y Riobamba, Zona 3 interandina, Ecuador
}

\section{Mining potential of two apiaries located in the Cevallos and Riobamba cantons, Inter-Andean Zone 3, Ecuador}

\author{
Miguel Ángel Guallpa Calva. ${ }^{1}$, Armando Esteban Espinoza Espinoza. ${ }^{2}$, Jorge Marcelo \\ Caranqui Aldaz. ${ }^{3} \&$ Edmundo Danilo Guilcapi Pacheco. ${ }^{4}$
}

\section{Resumen}

DOI: https://doi.org/10.33262/concienciadigital.v3i4.1.1469

Uno de los pilares para asegurar la producción apícola, es disponer de información confiable de la flora circundante y de los elementos que esta aporta al colmenar. Con ello, se puede planificar esquemas de manejo que incrementen la productividad. Frente a la necesidad de datos, se realizó el estudio de dos apiarios para contribuir al desarrollo apícola en la región interandina Zona 3, con el propósito de sustentar su gestión. Se priorizo identificar las especies vegetales de importancia melífera, y estimar su abundancia más la duración de la floración. Con la aplicación de los métodos: documental, y de campo, para el reconocimiento taxonómico de plantas melíferas, su cantidad, más el tiempo que persiste en fase de florecimiento de cada especie apícola evaluada, y la sistematización de los datos recopilados. Para el área del apiario A, se reporta 38 especies vegetales pertenecientes a 22 familias botánicas, siendo las más representativas: Asteraceae con 7 especies, y Rosaceae con 5. Por su parte, en el área del colmenar B, se registró 64 especies vegetales, correspondientes a 30 familias botánicas en las que se distingue la familia Fabaceae con 15 especies, y Asteraceae con 6. En abundancia para el apiario domina la especie Raphanus spp., en la subárea de rastrojos con más de 10000 individuos con flores, seguida por Prunus persica con 1000 a 10000 individuos con flores formando parte de la subárea de cultivos frutícolas y en el caso del apiario B, se destaca Raphanus spp con más de 10000 individuos con flores que predominan en la subárea de cultivos, y con menos de 1000 individuos en floridez para el

\footnotetext{
${ }^{1}$ Escuela Superior Politécnica de Chimborazo, Chimborazo-Riobamba, miguel.guallpa@espoch.edu.ec, https://orcid.org/0000-0001-5392-036X

${ }^{2}$ Escuela Superior Politécnica de Chimborazo, Chimborazo-Riobamba, armando.espinoza@espoch.edu.ec, https://orcid.org/0000-0002-8566-6594

3 Escuela Superior Politécnica de Chimborazo, Chimborazo-Riobamba, jcaranqui@espoch.edu.ec, https://orcid.org/0000-0001-7555-1294

${ }^{4}$ Investigador Independiente, Chimborazo-Riobamba, daniloguilcapi@gmail.com, https://orcid.org/0000-0002-2876-4702
} 
resto de los especímenes. Cuya duración del florecimiento brinda una oferta parcialmente continua de recursos florales entre tres a seis meses, a excepción del área de pastos.

Palabras claves: Especies melíferas, néctar, polen, apiario

\begin{abstract}
.
One of the pillars to ensure beekeeping production is to have reliable information on the surrounding flora and the elements that it contributes to the apiary. With this, it is possible to plan management schemes that increase productivity. Faced with the need for data, a study of two apiaries was carried out to contribute to the development of beekeeping in the interAndean region Zone 3, in order to support their management. It was prioritized to identify the plant species of melliferous importance, and to estimate their abundance plus the duration of flowering. With the application of methods: documentary, and field, for the taxonomic recognition of honey plants, their quantity, plus the time that each bee species persists in the flowering phase, test, and the systematization of the collected data. For the area of apiary A, 38 plant species belonging to 22 botanical families are reported, the most representative being: Asteraceae with 7 species, and Rosaceae with 5. On the other hand, in the area of apiary B, 64 plant species were recorded, corresponding to 30 botanical families in which the Fabaceae family is distinguished with 15 species, and Asteraceae with 6. In abundance for the apiary the species Raphanus spp. dominates, in the stubble subarea with more than 10,000 individuals with flowers, followed by Prunus persica with 1000 to 10000 individuals with flowers forming part of the sub-area of fruit crops and in the case of apiary B, Raphanus spp stands out with more than 10,000 individuals with flowers that predominate in the subarea of crops, and with less than 1000 individuals in floridity for the rest of the specimens. Whose duration of flowering provides a partially continuous supply of floral resources between three to six months, an exception of the pasture area.
\end{abstract}

Keywords: Honey species, nectar, pollen, apiary

\title{
Introducción
}

Los emprendimientos apícolas en los cantones Cevallos y Riobamba, muestran progresos importantes debido a procesos tecnológicos aplicados por los apicultores orientadas a obtener una producción sostenible, misma que tiene relación con el mayor apoyo brindado por parte del gobierno central hacia este sector de la agricultura, es así que desde el año 2014, el Ministerio de Agricultura, Ganadería, Acuacultura y Pesca (MAGAP) reactivó el Programa Nacional de Apicultura (PRONAPIS) para promover la expansión de la producción doméstica. Con un rendimiento promedio de miel de 10,2 kg por colmena al año, pero, en caso de aplicarse el esquema de manejo del MAGAP, su productividad aumentaría a 15,2 kilogramos por colmena por año.

La producción apícola es una actividad que aprovecha la vegetación, tanto en su estado natural como modificado, su disponibilidad representa el recurso con que cuentan las abejas para alimentarse y producir (Montoya, Baca, \& Bonilla, 2017; Ocaña \& Ocaña, 2008). 
Siendo fundamental tener un adecuado conocimiento de la oferta floral como pilar requerido para orientar la producción, diferenciar productos de la colmena y mejorar la competitividad (Silva \& Restrepo, 2012).

Como aporte del proyecto Impulso a la producción apícola a partir de la constitución de sistemas agroecológicos, de responsabilidad del grupo de investigación denominado "Conservación y Producción sustentable de los Recursos Naturales y Entomológicos (COPROSURENE)" de la Escuela Superior Politécnica de Chimborazo (ESPOCH). En respuesta a la necesidad de conocer los elementos florísticos y los períodos de floración de las plantas apícolas circundantes a cada colmenar, la generación de información preliminar, es indispensable para la toma de decisiones de manejo de esos componentes melíferos, que inciden en cambios en el flujo de néctar y polen a las colmenas.

En este trabajo se estudian las plantas de interés apícola que integran áreas adyacentes a dos apiarios, uno en el predio "Macají de la Facultad de Recursos Naturales-ESPOCH, y otro situado en la estación experimental Querochaca-UTA, las dos unidades productivas poseen sistemas agroecológicos, que contribuyen al paisaje, a la protección del equilibrio del ambiente, además el proveer de espacios para la ejecución de prácticas y trabajos de investigación (Guallpa et al., 2019).

La identificación de la flora melífera es muy requerida para la gestión de este valioso recurso a fin de promover el desarrollo de la apicultura local y regional. Basándose en observaciones de las visitas de abejas, la distribución de plantas apícolas y su abundancia en las superficies de investigación, complementado con el monitoreo del periodo de floración, accesibilidad a la flor que tienen las abejas en cada tipo de vegetación por predio, son elementos de manejo y de interés económico claves en esta actividad agropecuaria (Sánchez, 2013). Por lo expuesto, se propuso identificar las especies vegetales de importancia melífera, y estimar su abundancia más la duración de la floración en las dos áreas de estudio.

\section{Materiales y Métodos}

Esta investigación consideró la evaluación de múltiples sistemas agroecológicos que forman parte de los predios de la Estación Experimental Querochaca UTA y Facultad de Recursos Naturales ESPOCH, el primero se denomina A, ubicado en el kilómetro 2 vía Quero, Cantón Cevallos, Provincia de Tungurahua, el lugar está dentro de la formación ecológica, Matorral húmedo montano, sector norte y centro de los valles interandinos, subregión norte y centro. El segundo se le nombra B, se localiza a $2 \mathrm{~km}$ de la ciudad de Riobamba, Provincia de Chimborazo. El área se encuentra dentro de la formación: estepa espinosa Montano Bajo (Sierra et al., 1999).

Geográficamente se ubican en los puntos de referencia: UTM Zona 17S Datum WGS 84, y las principales variables climáticas son (Tabla 1). 
Tabla 1. Ubicación geográfica y variables climáticas de las áreas del estudio

\begin{tabular}{ccccccc}
\hline Apiarios & $\mathrm{X}$ & $\mathrm{Y}$ & $\begin{array}{c}\text { Altitud } \\
(\mathrm{msnm})\end{array}$ & $\begin{array}{c}\text { Temperatura } \\
{ }^{\circ} \mathrm{C}\end{array}$ & $\begin{array}{c}\text { Precipitación } \\
\mathrm{mm}\end{array}$ & $\begin{array}{c}\text { Humedad relativa } \\
\end{array}$ \\
& & & & & & $\%$ \\
A & 766050 & 9846966 & 2890 & $13,2^{\circ}$ & 603 & $75,8 \%$ \\
B & 757989 & 9817208 & 2840 & $13,3^{\circ} \mathrm{C}$ & 560,1 & $85 \%$ \\
\hline
\end{tabular}

Fuentes: Estación Meteorológica Querochaca (2018), y Estación Meteorológica de la ESPOCH; serie 1984-2014

\section{Métodos}

Por el tipo investigación se aplicó los métodos: documental y de campo, utilizados para el reconocimiento de plantas apícolas y duración de la floración (Calvache, 2016). La valoración de las plantas melíferas tuvo lugar durante el tercer y cuarto trimestre del año 2019, y en el caso del registro de abundancia se realizó cada 30 días de acuerdo con el periodo de evaluación considerado en el estudio, y con la ejecución de las siguientes actividades:

\section{Delimitación de las áreas evaluadas}

Se realizó mediante dos visitas de campo a los sitios en estudio, el registro de coordenadas y cálculo de áreas con GPS, más la categorización de las subáreas de acuerdo con los usos de la tierra que posee cada predio, se determinó la distribución de la superficie evaluada que se indica en la Tabla 2, al utilizar el guion de observación directa elaborada por Guallpa, Guilcapi \& Espinoza. (2019).

Tabla 2. Distribución de usos de suelo en cada apiario

\begin{tabular}{|c|c|c|c|c|}
\hline \multirow{2}{*}{$\begin{array}{l}\text { Apiarios } \\
\text { Categoría }\end{array}$} & \multicolumn{2}{|l|}{$\mathbf{A}$} & \multicolumn{2}{|l|}{ B } \\
\hline & Áreas (Ha) & $\%$ & Áreas (Ha) & $\%$ \\
\hline Bosques plantados & 3,1 & 24,9 & 8 & 32 \\
\hline Arbustos & 0,3 & 2,5 & 1 & 4 \\
\hline Rastrojo & 1,7 & 14,2 & 1 & 4 \\
\hline Pastos & & & 2 & 8 \\
\hline Cultivos & 1,9 & 15,9 & 2 & 8 \\
\hline Sistemas agroforestales & 1 & 8,4 & 2 & 8 \\
\hline Bordes de caminos & 4,2 & 33,8 & 2 & 8 \\
\hline Ornamental & & & 7 & 28 \\
\hline Total & 12,2 & 100 & 25 & 100 \\
\hline
\end{tabular}

Fuente: Guión de observación directa aplicada (Guallpa, Guilcapi \& Espinoza, 2019

\section{Monitoreo e identificación taxonómica de especies vegetales}


Sobre la base de los usos del suelo de cada predio, el monitoreo con el muestreo por observación de la vegetación presente integrando congregados o elementos florísticos alrededor de los dos colmenares.

La identificación de los elementos florísticos con potencial melífero se realizó en la cuarta y quinta visita a las dos áreas evaluadas, al distinguir aquellas que son frecuentadas por las abejas en virtud de la oferta de néctar y/o polen, mediante el reconocimiento de características diagnósticas de los especímenes en campo, más su verificación utilizando bibliografía especializada de Aguirre (2018); Rivas (2017); Palacios (2016); Aguirre, Yaguana \& Merino (2014); Aguirre \& Yaguana, (2013). Se registró las especies con potencial melífero, al observar si se encontraban en floración, el recurso ofertado y la forma de detectar por las abejas; el néctar, polen o la combinación néctar y polen, al utilizar el protocolo de monitoreo de dicha oferta, la cual fue comparada con los resultados obtenidos en los trabajos de Aguirre \& Castañeda (2015); Silva \& Restrepo (2012).

\section{Determinación de la abundancia de la flora melífera}

Se empleó el esquema semi-cuantitativo elaborado por May \& Rodríguez. (2012), quienes en su estudio establecieron un valor de 1 a 5 , para estimar la abundancia, mediante la escala: (1) menos de 1000 individuos con floridez dentro del perímetro de actividad de las abejas, cubriendo menos de $1 \%$ del área, (2) de 1000 a 10000 individuos con floridez dentro del perímetro de actividad de las abejas, cubriendo menos de 1\% del área, (3) más de 10000 individuos con floridez dentro del perímetro de actividad de las abejas, cubriendo menos de $1 \%$ del área, (4) individuos con floridez dentro del perímetro de actividad de las abejas cubren entre $1 \%$ y $5 \%$ del área, y (5) individuos con floridez dentro del perímetro de actividad de las abejas cubren más de $5 \%$ del área.

Finalmente, se asignan valores de 1 a 5 para representar la duración de la floración de cada una de las especies melíferas (DF; Tabla 3).

Tabla 3. Escala del índice duración de la floración (DF)

\begin{tabular}{cl}
\hline índice DF & \multicolumn{1}{c}{ Descripción } \\
\hline 1 & De un mes o menos \\
2 & Entre 2 o 3 meses \\
3 & Entre 4 o 5 meses \\
4 & Entre 6 o 7 meses \\
5 & Entre 8 - 12 meses \\
\hline
\end{tabular}

\section{Sistematización de la información}

Para el registro, organización, sistematización y análisis de datos de plantas melíferas, su abundancia y duración de la floración, se utilizó la hoja de cálculo de Excel.

\section{Resultados y Discusión}

\section{A. Plantas melíferas del apiario A}

Se obtuvo un listado de 38 elementos florísticos pertenecientes a 22 familias botánicas, para el apiario A, siendo la familia de plantas con mayor cantidad de especímenes reportados 
corresponde a Asteraceae con 7, Rosaceae con 5, Fabaceae, Myrtaceae, Solanaceae con 3 y, las demás familias botánicas están representadas por un especímen único (Tabla 4). Estos valores a nivel de familias concuerdan de forma parcial con investigaciones similares que indican a Fabaceae y Asteraceae como las predominantes en la parroquia Licto, al comparar con la flora melífera para un apiario situado a 2735 m.s.n.m en el estudio desarrollado por Guallpa, Guilcapi, \& Espinoza (2019). En otra investigación, la familia Asteraceae fue reportada como una de las más importantes al evaluar tres apiarios en la zona rural de Popayán que se localiza a una altitud promedio de 1738 m.s.n.m. (Vivas, Maca \& Pardo, 2008).

Tabla 4. Listado de plantas nectaríferas y poliníferas en el apiario A

\begin{tabular}{|c|c|c|c|c|c|}
\hline \multirow[t]{2}{*}{$\mathbf{N}^{\circ}$} & \multirow[t]{2}{*}{ Familia } & \multirow[t]{2}{*}{ Nombre científico } & \multirow[t]{2}{*}{ Nombre común } & \multicolumn{2}{|c|}{$\begin{array}{l}\text { Recurso } \\
\text { ofertado }\end{array}$} \\
\hline & & & & Néctar & Polen \\
\hline 1 & Amaranthaceae & Alternanthera porrigens Jacq. & Moradilla & & $\bullet$ \\
\hline 2 & Araliaceae & Hedera helix $\mathrm{L}$. & Hiedra & • & • \\
\hline 3 & Asteraceae & Artemisia absinthium L. & Ajenjo & & $\bullet$ \\
\hline 4 & Asteraceae & Baccharis latifolia Ruiz \& Pav. & Chilca & $\bullet$ & $\bullet$ \\
\hline 5 & Asteraceae & Taraxacum officinale Weber & Diente de león & • & - \\
\hline 6 & Asteraceae & Ambrosia arborescens Mill & Marco & & • \\
\hline 7 & Asteraceae & Leucanthemum vulgare Lam. & Margarita & - & • \\
\hline 8 & Asteraceae & Galinsoga parviflora $\mathrm{Cav}$. & Pacunga & & • \\
\hline 9 & Asteraceae & Matricaria chamomilla $\mathrm{L}$ & Manzanilla & • & • \\
\hline 10 & Betulaceae & Alnus acuminata Kunth & Aliso & & • \\
\hline 11 & Bignoniaceae & Tecoma stans (L.) Juss. & Cholán & • & • \\
\hline 12 & Brassicaceae & Raphanus spp. & Falso nabo lila & $\bullet$ & • \\
\hline 13 & Cupresaceae & Cupressus sempervirens $\mathrm{L}$. & Ciprés & & • \\
\hline 14 & Fabaceae & Otholobium mexicanum (L. f.) J.W. Grimes & Chanchilva & $\bullet$ & \\
\hline 15 & Fabaceae & Trifolium repens $\mathrm{L}$. & Trébol & • & • \\
\hline 16 & Fabaceae & Spartium junceum L. & Retama & & • \\
\hline 17 & Geraniaceae & Pelargonium spp. & Geranio & • & • \\
\hline 18 & Lamiaceae & Lavandula angustifolia Mill. & Lavanda & • & • \\
\hline 19 & Mimosaceae & Acacia macracantha Humb & Acacia & • & • \\
\hline 20 & Myrtaceae & Callistemon citrinus (Curtis) Skeels & Cepillo rojo & • & • \\
\hline 21 & Myrtaceae & Callistemon viminalis (Sol. ex Gaertn.) G. Don & Cepillo blanco & • & • \\
\hline 22 & Myrtaceae & Eucalyptus globulus Labill & Eucalipto & $\bullet$ & • \\
\hline 23 & Myricaceae & $\begin{array}{l}\text { Morella pubescens (Humb. et Bonpl.ex Willd.) } \\
\text { Wilbur }\end{array}$ & Laurel de cera & • & • \\
\hline 24 & Oxalidaceae & Oxalis tuberosa Molina & Oca & & • \\
\hline 25 & Pinaceae & Pinus radiata D. Don & Pino & & • \\
\hline 26 & Poaceae & Zea mays L. & Maíz & & $\bullet$ \\
\hline 27 & Rosaceae & Pyrus communis L. & Pera & $\bullet$ & • \\
\hline 28 & Rosaceae & Prunus persica L. & Durazno & • & • \\
\hline 29 & Rosaceae & Prunus serotina Ehrh & Capulí & $\bullet$ & • \\
\hline 30 & Rosaceae & Rubus glaucus Benth. & Mora & $\bullet$ & $\bullet$ \\
\hline 31 & Rosaceae & Rosa sp. & Rosa roja & $\bullet$ & • \\
\hline
\end{tabular}


ISSN: 2600-5859

\begin{tabular}{|c|c|c|c|c|c|}
\hline 32 & Rutaceae & Citrus limon $\mathrm{L}$. & Limón rosa & - & • \\
\hline 33 & Salicaceae & Salix humboldtiana Willd. & Sauce & - & • \\
\hline 34 & Scrophulariaceae & Buddleja incana (Ruiz \& Pav.) & Quishuar & - & \\
\hline 35 & Solanaceae & Solanum betaceum Cav. & Tomate de árbol & & - \\
\hline 36 & Solanaceae & Solanum tuberosum $\mathrm{L}$. & Papa & & • \\
\hline 37 & Solanaceae & Solanum nigrescens $\mathrm{M}$. Martens \& Galeotti & Hierba mora & - & • \\
\hline 38 & Verbenaceae & Verbena officinalis $\mathrm{L}$. & Verbena & - & • \\
\hline
\end{tabular}

Del total de especies identificadas en la investigación, 24 son especies productoras de néctar y polen, mientras que 12 especies ofertan polen y tan solo dos especies proveen néctar (Tabla 4).

\section{Plantas melíferas del apiario B}

Se registró un listado de 64 elementos florísticos pertenecientes a 30 familias botánicas para el colmenar B, siendo la familia con mayor cantidad de especies reportadas, Fabaceae con 15 especies. Se destaca también por el número de especies las familias: Asteraceae con $6 \mathrm{y}$ Myrtaceae con 4, seguida por las familias; Cactaceae, Rosaceae y Solanaceae con tres especies. Existen 18 familias botánicas representadas por una especie única (Tabla 5). Los resultados de las familias predominantes concuerdan con un estudio similar que reporta a Fabaceae y Asteraceae como las más importantes, no así en cuanto al número de ejemplares, superando con 27 en referencia a los 37 especímenes, según el reporte obtenido al caracterizar la flora melífera circundante a un apiario en la parroquia Licto, ubicado a una altitud de 2735 msnm y cuya área se encuentra dentro de similar formación ecológica (Guallpa, Guilcapi, \& Espinoza, 2020).

Tabla 5. Resumen de plantas nectaríferas y poliníferas presentes en el Apiario B

\begin{tabular}{|c|c|c|c|c|c|}
\hline \multirow[t]{2}{*}{$\mathbf{N}^{\circ}$} & \multirow[t]{2}{*}{ Familia } & \multirow[t]{2}{*}{ Nombre científico } & \multirow[t]{2}{*}{ Nombre común } & \multicolumn{2}{|c|}{$\begin{array}{l}\text { Recurso } \\
\text { ofertado }\end{array}$} \\
\hline & & & & Néctar & Polen \\
\hline 1 & Asparagaceae & Agave americana $\mathrm{L}$. & Cabuya negra & • & • \\
\hline 2 & Asparagaceae & Furcraea andina Trel. & Cabuya blanca & • & $\bullet$ \\
\hline 3 & Anacardiaceae & Schinus molle L. & Molle & • & - \\
\hline 4 & Araliaceae & Oreopanax ecuadorensis Seem. & Pumamaki & - & \\
\hline 5 & Asteraceae & Taraxacum officinale Weber & Diente de león & • & • \\
\hline 6 & Asteraceae & Baccharis latifolia (Ruiz \& Pav.) Pers. & Chilca & • & • \\
\hline 7 & Asteraceae & Bidens andicola $\mathrm{L}$. & Ñachag & • & • \\
\hline 8 & Asteraceae & Galinsoga ciliata (Raf.) S.F. Blake & Amor seco & & - \\
\hline 9 & Asteraceae & Gnaphalium spp & Gordolobo & & • \\
\hline 10 & Asteraceae & Sonchus oleraceus L. & Cerraja & & • \\
\hline 11 & Betulaceae & Alnus acuminata Kunth & Aliso & & • \\
\hline 12 & Bignoniaceae & Tecoma stans (L.) Juss. & Cholán & - & - \\
\hline 13 & Bignoniaceae & Jacaranda mimosifolia D. Don & Jacaranda & • & $\bullet$ \\
\hline 14 & Brassicaceae & Raphanus spp. & Falso nabo & • & - \\
\hline
\end{tabular}


ISSN: 2600-5859

\begin{tabular}{|c|c|c|c|c|c|}
\hline 15 & Brassicaceae & Brassica oleracea var. italica & Brócoli & - & - \\
\hline 16 & Cactaceae & Opuntia ficus-indica (L.) Mill. & Tuna & - & • \\
\hline 17 & Cactaceae & Cleistocactus sepium Kunth & Cactus & $\cdot$ & • \\
\hline 18 & Cactaceae & Opuntia cylindrica (Lam.) DC. & Cactus & $\cdot$ & • \\
\hline 19 & Caprifoliaceae & Sambucus nigra $\mathrm{L}$. & Sauco & $\cdot$ & • \\
\hline 20 & Cucurbitaceae & Cucumis sativus $\mathrm{L}$. & Pepinillo & & • \\
\hline 21 & Euphorbiaceae & Ricinus communis $\mathrm{L}$. & Higuerilla & $\cdot$ & \\
\hline 22 & Fabaceae & Acacia retinodes Schltdl. & Acacia & & • \\
\hline 23 & Fabaceae & Acacia dealbata Link & Acacia fina & - & • \\
\hline 24 & Fabaceae & Acacia melanoxylon R.Br. & Acacia & $\cdot$ & $\cdot$ \\
\hline 25 & Fabaceae & Inga spp & Guaba & $\cdot$ & • \\
\hline 26 & Fabaceae & Caesalpinia spinosa (Molina) Kuntze & Guarango & - & • \\
\hline 27 & Fabaceae & Pisum sativum $\mathrm{L}$. & Arveja & $\cdot$ & $\cdot$ \\
\hline 28 & Fabaceae & Trifolium repens $\mathrm{L}$. & Trébol blanco & $\cdot$ & $\cdot$ \\
\hline 29 & Fabaceae & Medicago lupulina $\mathrm{L}$. & Lupulina & $\cdot$ & • \\
\hline 30 & Fabaceae & Melilotus indicus (L.) All. & Meliloto & & $\cdot$ \\
\hline 31 & Fabaceae & Lupinus mutabilis Sweet. & Chocho & $\cdot$ & • \\
\hline 32 & Fabaceae & Genista monspessulana (L) L. A. S & Lupina & & • \\
\hline 33 & Fabaceae & Spartium junceum L. & Retama & & $\cdot$ \\
\hline 34 & Fabaceae & Mimosa quitensis Benth. & Faique & $\cdot$ & • \\
\hline 35 & Fabaceae & Paraserianthes lophantha (Willd.) & Acacia plumosa & $\cdot$ & • \\
\hline 36 & Fabaceae & Dalea coerulea L.f. & Iso & $\cdot$ & • \\
\hline 37 & Juglandaceae & Juglans neotropica Diels. & Nogal & $\cdot$ & • \\
\hline 38 & Lamiaceae & Rosmarinus officinalis L. & Romero & • & \\
\hline 39 & Meliaceae & Cedrela montana Moritz & Cedro & $\cdot$ & $\cdot$ \\
\hline 40 & Moraceae & Morus alba $\mathrm{L}$. & Morera & & • \\
\hline 41 & Myrtaceae & Eucalyptus globulus Labill & Eucalipto & - & • \\
\hline 42 & Myrtaceae & Eucalyptus citriodora Hook & Eucalipto aromático & $\cdot$ & • \\
\hline 43 & Myrtaceae & $\begin{array}{l}\text { Callistemon viminalis (Sol. ex Gaertn.) } \\
\text { G. Don }\end{array}$ & Cepillo blanco & & $\bullet$ \\
\hline 44 & Myrtaceae & Melaleuca citrina Turcz. & Cepillo rojo & & • \\
\hline 45 & Oleaceae & Ligustrum japonicum Thunb. & Azhares & $\cdot$ & $\cdot$ \\
\hline 46 & Papaveraceae & Argemone mexicana $\mathrm{L}$. & Cardo santo & & • \\
\hline 47 & Poaceae & Zea mays $\mathrm{L}$. & Maíz & & • \\
\hline 48 & Polygonaceae & Rumex obtusifolius L. & Lengua de vaca & $\cdot$ & • \\
\hline 49 & Plantaginaceae & Plantago lanceolata $\mathrm{L}$. & Llánten & & • \\
\hline 50 & Proteaceae & Grevillea robusta A. Cunn. ex $R . B r$. & Árbol del fuego & $\cdot$ & $\cdot$ \\
\hline 51 & Rosaceae & Prunus serotina Ehrh & Capulí & $\cdot$ & • \\
\hline 52 & Rosaceae & Rubus glaucus Benth & Mora silvestre & $\cdot$ & • \\
\hline 53 & Rosaceae & Eriobotrya japonica (Thunb.) Lindl. & Níspero & $\cdot$ & • \\
\hline 54 & Rutaceae & Citrus spp & Limonero & $\cdot$ & • \\
\hline 55 & Rutaceae & Ruta graveolens $\mathrm{L}$. & Ruda & • & • \\
\hline 56 & Salicaceae & Salix humboldtiana Willd. & Sauce & $\cdot$ & $\cdot$ \\
\hline 57 & Salicaceae & Populus deltoides Bartram ex Marshall & Álamo & $\cdot$ & $\cdot$ \\
\hline 58 & Sapindaceae & Dodonaea viscosa Jacq. & Chamana & • & • \\
\hline
\end{tabular}


ISSN: 2600-5859

\begin{tabular}{|c|l|l|l|c|c|}
\hline 59 & Solanaceae & Physalis peruviana L. & Uvilla & $\bullet$ & \\
\hline 60 & Solanaceae & $\begin{array}{l}\text { Solanum nigrescens M. Martens \& } \\
\text { Galeotti }\end{array}$ & Hierba mora & & \\
\hline 61 & Solanaceae & Datura stramonium L. & Chamico & $\bullet$ & \\
\hline 62 & Scrophulariaceae & Buddleja incana (Ruiz \& Pav.) & Quishuar & $\bullet$ & \\
\hline 63 & Scrophulariaceae & Buddleja bullata Kunth & Quishuar & $\bullet$ & \\
\hline 64 & Verbenaceae & $\begin{array}{l}\text { Lantana megapotamica } \text { (Spreng.) } \\
\text { Tronc. }\end{array}$ & Lantana morada & & \\
\hline
\end{tabular}

Del total de especies identificadas, 7 corresponden a productoras de néctar, 15 de polen y 42 especies ofertan tanto polen como néctar. Este trabajo indica una mayor disponibilidad de recursos florísticos apícolas que pueden ser aprovechadas por las abejas a especímenes vegetales pertenecientes a las familias Fabaceae y Asteraceae, porque les proporcionan en la mayoría de los casos, polen y néctar (Pinilla, \& Nates, 2015). Con base en los resultados obtenidos, las plantas con potencial apícola son datos importantes a tener en cuenta en la planificación de la actividad apícola (Insuasty, Martínez, \& Jurado, 2016) puesto que es la materia prima de la cual las abejas recolectan los recursos que utilizan para la elaboración de su alimento, planificar las diferentes labores en la colmena, y permite promover el manejo de la cobertura vegetal para contribuir al mejoramiento de las condiciones técnicas de cada apiario (Doke, Frazier, \& Grozinguer, 2015).

\section{Abundancia y duración de floración de especies vegetales del apiario A}

En referencia a las unidades de uso del suelo del colmenar A como muestra la Tabla 6 de abundancia y floración de plantas apícolas en las áreas adyacentes al colmenar en la área de rastrojos, se estimo más de 10000 individuos y en el área de cultivos con menos de 1000 plantas con floridez de Raphanus spp., para el resto de los especímenes evaluados con menos de 1000 individuos con flores, cuya ocupación del área total, está integrada varios sistemas de uso del suelo, mismos que brindan recursos apícolas, cuya duración del florecimiento ocurrió de entre tres a seis meses correspondiente al segundo semestre del año 2019, a excepción del área de pastos.

Tabla 6. Abundancia y floración de plantas apícolas en las áreas del colmenar A

\begin{tabular}{|c|c|c|c|c|c|c|c|c|c|c|}
\hline Nombre científico & 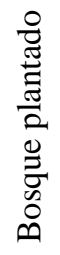 & 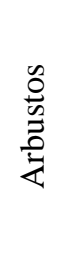 & 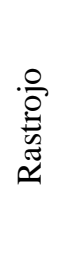 & $\frac{\tilde{0}}{\tilde{0}}$ & $\stackrel{0}{0}$ & 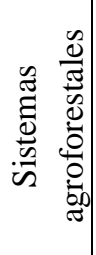 & 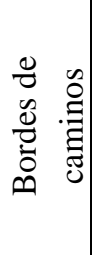 & 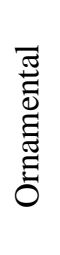 & $\begin{array}{c}\text { Meses } \\
\text { J-A-S }\end{array}$ & $\begin{array}{l}\text { Meses } \\
\text { O-N-D }\end{array}$ \\
\hline Alternanthera porrigens & & & & & & & 1 & & & \\
\hline Hederea helix & & & & & & 1 & & & & \\
\hline Artemisia absinthium & & & 1 & & 1 & & & & & \\
\hline Baccharis latifolia & & 1 & & & & & 1 & & & \\
\hline Taraxacum officinale & & & 1 & & & & 1 & & & \\
\hline Ambrosia arborescens & & 1 & & & & & 1 & & & \\
\hline
\end{tabular}




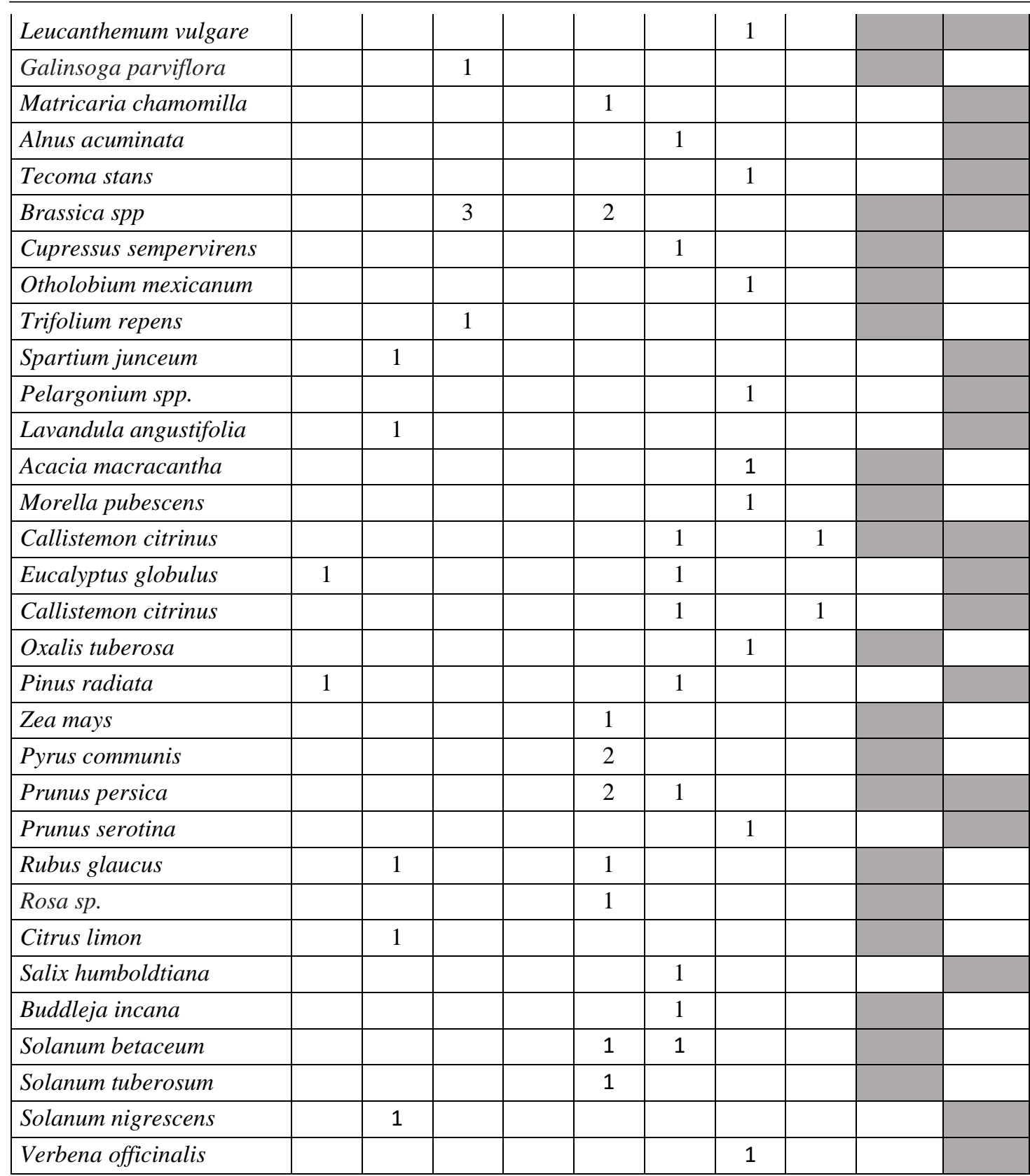

Abundancia: (1) menos de 1000 individuos con floridez dentro del perímetro de actividad de las abejas, (2) de 1000 a 10000 individuos con floridez dentro del perímetro de actividad de las abejas, cubriendo menos de $1 \%$ del área y (3) más de 10000 individuos con floridez dentro del perímetro de actividad de las abejas.

De acuerdo con los datos obtenidos para el apiario A, se demuestra que hay una oferta continua de recursos nectaríferos y poliníferos disponibles y las abejas puedan usar para obtener una gama de tipos y calidades de miel de abeja (Zavala et al., 2013). Cuya disponibilidad indica una duración de la florescencia entre 3 a 6 meses dependiendo de la fase de crecimiento y desarrollo de cada una de los especímenes valorados en esta investigación.

\section{Abundancia y duración de floración de especies vegetales del apiario $\mathbf{B}$}

En relación a las unidades de uso del suelo del Apiario B, según la tabla 7 en el área de cultivo, se estimo más de 10000 individuos con floridez de Raphanus spp., y menos de 1000 
individuos con floridez presentes, para el resto de especímenes reportados, cuya ocupación se distribuye en los siete sistemas de uso del suelo, a excepción del área de pastos.

Tabla 7. Abundancia y floración de plantas apícolas en las áreas del colmenar B

\begin{tabular}{|c|c|c|c|c|c|c|c|c|c|c|}
\hline Nombre científico & 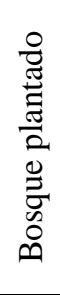 & 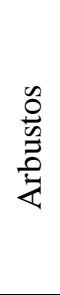 & 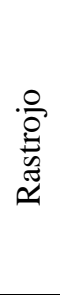 & $\begin{array}{l}\tilde{0} \\
\tilde{a} \\
\tilde{a}\end{array}$ & $\frac{\stackrel{n}{Z}}{\Xi}$ & 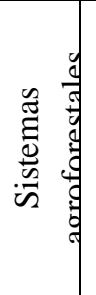 & 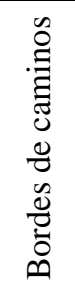 & 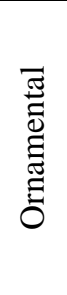 & $\begin{array}{c}\text { Meses } \\
\text { J-A-S }\end{array}$ & $\begin{array}{l}\text { Meses } \\
\text { O-N-D }\end{array}$ \\
\hline Agave americana & & & 1 & & & & & & & \\
\hline Furcraea andina & & & 1 & & & & & & & \\
\hline Schinus molle & 1 & & & & & & & 1 & & \\
\hline Oreopanax ecuadorensis & 1 & & & & & & & 1 & & \\
\hline Taraxacum officinale & & & 1 & & & & & & & \\
\hline Baccharis latifolia & 1 & & 1 & & & & & & & \\
\hline Bidens andicola & & & 1 & & & & & & & \\
\hline Galinsoga ciliata & & & & & 1 & & & & & \\
\hline Gnaphalium spp & & & & & & & & 1 & & \\
\hline Sonchus oleraceus & & & 1 & & & & & & & \\
\hline Alnus acuminata & 1 & & & & & & & & & \\
\hline Tecoma stans & 1 & & & & & & & 1 & & \\
\hline Jacaranda mimosifolia & & & & & & & & 1 & & \\
\hline Raphanus spp. & & & 1 & & 3 & & & & & \\
\hline Brassica oleracea var. Italica & & & & & 1 & & & & & \\
\hline Opuntia ficus-indica & 1 & & & & & & & & & \\
\hline Cleistocactus sepium & 1 & & & & & & & & & \\
\hline Opuntia cylindrica & 1 & & 1 & & & & & & & \\
\hline Sambucus nigra & 1 & & & & & & & 1 & & \\
\hline Cucumis sativus & & & & & 1 & & & & & \\
\hline Ricinus communis & & & 1 & & & & & & & \\
\hline Acacia retinodes & 1 & & & & & & & & & \\
\hline Acacia dealbata & 1 & & & & & & & & & \\
\hline Acacia melanoxylom & 1 & & & & & & & 1 & & \\
\hline Inga spp & 1 & & & & & & & & & \\
\hline Caesalpinia spinosa & 1 & & & & & & & 1 & & \\
\hline Pisum sativum & & & & & 1 & & & & & \\
\hline Trifolium repens & & & 1 & & & & & & & \\
\hline Medicago lupulina & & & & & & & 1 & & & \\
\hline Melilotus indicus & & & & & & & 1 & & & \\
\hline Lupinus mutabilis & & & 1 & & & & & & & \\
\hline Genista monspessulana & & & & & & & & 1 & & \\
\hline Spartium junceum & & & 1 & & & & & & & \\
\hline
\end{tabular}




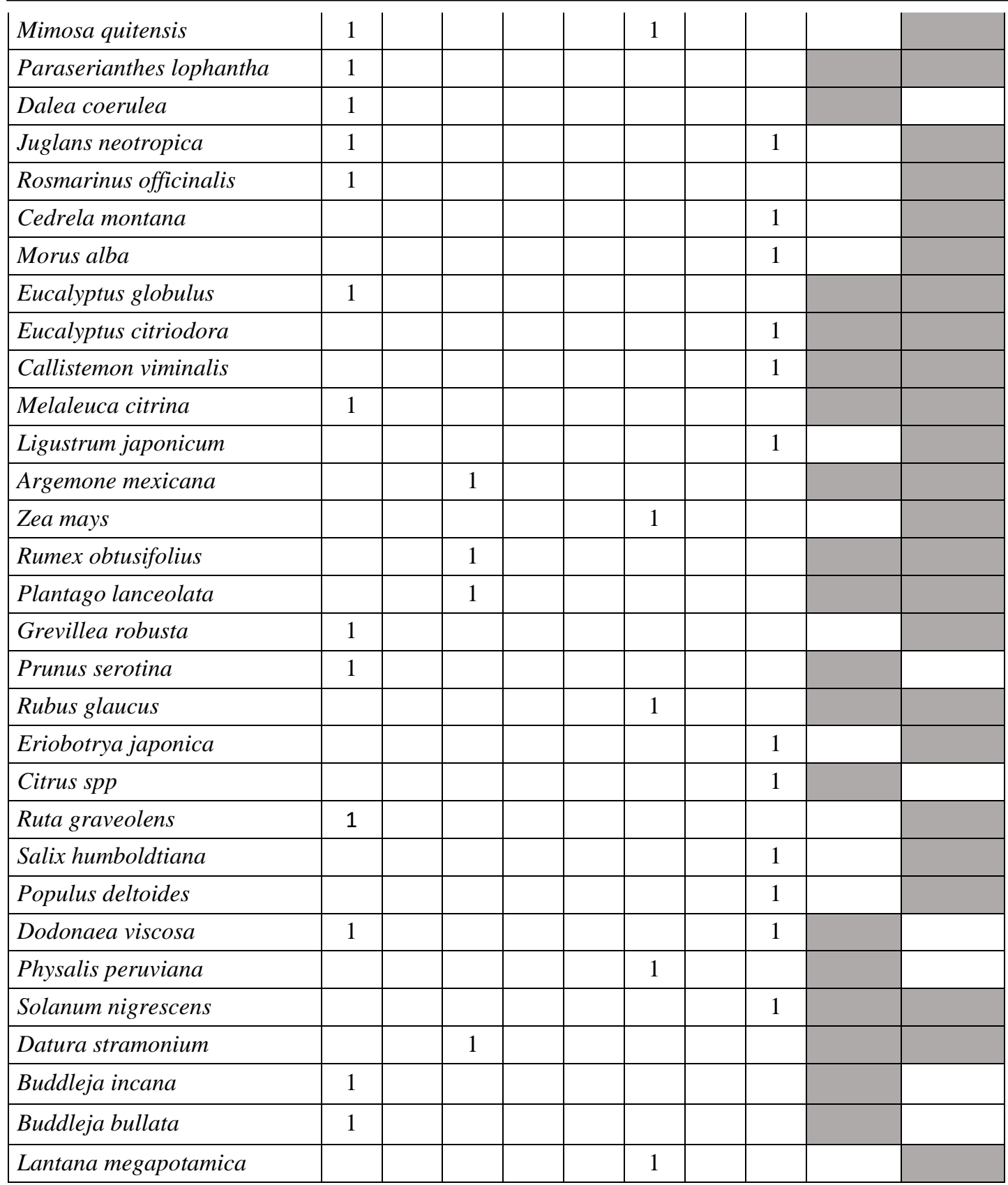

Abundancia: (1) menos de 1000 individuos con floridez dentro del perímetro de actividad de las abejas, (2) de 1000 a 10000 individuos con floridez dentro del perímetro de actividad de las abejas, cubriendo menos de $1 \%$ del área, y (3) más de 10000 individuos con floridez dentro del perímetro de actividad de las abejas.

Del análisis de abundancia de especies melíferas en los dos apiarios, para los sistemas de uso rastrojos y cultivos respectivamente, se destaca la predominancia de Raphanus spp, especie apícola en común y esto se debe a que son predios caracterizados por una alta actividad agrícola y por ende rotación de cultivos en ciertas áreas, son atributos que influyen en la disponibilidad de recursos melíferos, donde las abejas realizan selección según calidad, cantidad y accesibilidad, desplazándose a diferentes distancias, direcciones y coberturas vegetales (Nates,2016). En el caso del colmenar B, la duración de la florescencia fluctúa entre 3 a 6 meses variando en función del espécimen, su etapa de crecimiento y características de sistema de uso del suelo evaluado. 
La disponibilidad de información técnica facilitará el manejo de los apiarios. Dado que, la flora apícola está directamente influida por las condiciones climáticas, que definen su aporte a la colmena y determinan el comportamiento de la floridez (Brigitte, 2012).

\section{Conclusiones}

- Se obtuvo un listado de 38 especies con potencial melífero que representan a 22 familias botánicas, siendo las más representativas: Asteraceae con 7 especies, y Rosaceae con 5, que son útiles para Apis mellifera, en las subáreas que integran el apiario A, mientras que 64 especies melíferas para el apiario B, pertenecientes a 30 familias botánicas en las que se distingue la familia Fabaceae con 15 especies, y Asteraceae con 6.

- El monitoreo indica la preferencia de utilización del recurso floral a individuos la especie Raphanus spp, Prunus persica para el primer colmenar ocupando las subáreas de rastrojos y cultivos, en cambio en el segundo apiario dominan individuos de Raphanus spp pero en el área de cultivos, en ambos casos se evidencia el aporte como plantas de importancia para la colmena, la cobertura de arvenses con predominancia de vegetación herbácea de Raphanus spp.

- La ocupación del área total adyacente a los apiarios Ay B, brindan recursos melíferos diversos en casi la mayoría de sistemas de uso del suelo evaluados. Cuya duración del florecimiento de plantas apícolas refleja una oferta parcialmente continua de elementos florales oscilando entre tres a seis meses, a excepción del área de pastos. Así, mismo los datos obtenidos, son indicadores técnicos valiosos, que permitirán mejorar el manejo de los dos apiarios y de otros que posean condiciones técnicas similares

\section{Referencias Bibliográficas}

Aguirre, Z. (2018). Principios para el estudio de familias botánicas del Ecuador. Universidad Nacional de Loja. Loja, Ecuador.

Aguirre S., \& Castañeda, S. (2015). Identificación palinológica en mieles de la costa chica de Guerrero y Oaxaca. Tesis licenciatura. Unidad Académica de Medicina Veterinaria y Zootecnia, Universidad Autónoma de Guerrero, 84p.

Aguirre, Z., Yaguana, C., \& Merino, B. (2014). Plantas medicinales de la zona andina de la provincia de Loja. Primera Edición. Loja, Ecuador. 193 p.

Aguirre, Z., \& Yaguana, C. (2013). Árboles y arbustos de parques y avenidas de Loja. 
Calvache, J. (2016). La investigación científica como alternativa en la formación profesional. Colombia: CEPUN.

Doke, M., Frazier, M., \& Grozinguer, C. (2015). Overwintering Honey Bees: biolgy and management. Elsevier, 10,185-193.

Guallpa, M., Lara, N., Espinoza, M., Guilcapi, E., \& Fosado, O. (2019). Valoración cualitativa de una plantación de Eucalyptus globulus Labill en el sector de Licto, Riobamba, Ecuador. Polo del Conocimiento, 4(4), 126-152.

Guallpa, M., Guilcapi, E., \& Espinoza, A. (2019). Flora apícola de la zona estepa espinosa Montano Bajo, en la Estación Experimental Tunshi, Riobamba, Ecuador. Dominio de las Ciencias, 5(2), 71-93.

Guallpa, M., Guilcapi, E., \& Espinoza, A. (2020). Estimación de la flora melífera para la productividad apícola de la estación experimental Tunshi en el sector de Licto, Riobamba. Dominio de las Ciencias, 6(2), 181-202.

Insuasty, E., Martínez, J., \& Jurado, H. (2016). Identificación de flora y análisis nutricional de miel de abeja para la producción apícola. Biotecnología en el Sector Agropecuario y Agroindustrial. Biotecnología en el Sector Agropecuario y Agroindustrial, 14 (1), 37 44.

May, T., y Rodríguez, S. (2012). Plantas de interés apícola en el paisaje: Observaciones de campo y la percepción de apicultores en República Dominicana. Revista Geográfica de América Central, 48 (1), 133-162.

Ministerio de Agricultura, ganadería, acuacultura y pesca. MAGAP. (2014). Agrocalidad. Obtenido de http://www.agrocalidad.gob.ec/wp-content/uploads/2016/07/resolucion241.pdf

Montoya, B., Baca, A., \& Bonilla, B. (2017). Flora melífera y su oferta de recursos en cinco veredas del municipio de Piendamó, Cauca. Biotecnología en el Sector Agropecuario y Agroindustrial, 15(1), 20-28.

Ocaña, R., \& Ocaña, P. (2008). Prácticas de Apicultura. Servicio de Formación Agraria e Iniciativas. Junta de Castilla y León.

Palacios, W. (2016). Árboles del Ecuador: Especies representativas. Primera Edición. Universidad Técnica del Norte Ibarra, Ecuador

Pinilla, M., \& Nates, G. (2015). Diversidad de visitantes y aproximación al uso de nidos trampa para Xylacopa (Hymenoptera: Apidae) en una zona productora de pasiflora en Colombia. Actualidades Biológicas, 37(103), 143-153.

Rivas, J. (2017). Guía ilustrada de especies agrodiversas en el Ecuador. Segunda Edición. Universidad de Cuenca. Cuenca, Ecuador. 
Sánchez, C. (2013). Crianza y producción de abejas-apicultura. Perú: Ripalme.

Sierra, R., Cerón, C., Palacios, W., \& Valencia, R. (1999). Propuesta preliminar de un sistema de clasificación para el Ecuador Continental. Quito, Ecuador: Proyecto INEFAN/GEF-BIRF y EcoCiencia.

Silva, L; \& Restrepo, S. (2012). Flora Apícola. Determinación de la oferta floral apícola como mecanismo para optimizar la producción, diferenciar producto de la colmena y mejorar la competitividad. Instituto de investigación de recursos biológicos Alexander von Humboldt.

Vivas, N., Maca, J., \& Pardo, M. (2008). Caracterización cualitativa del polen recolectado por Apis mellifera $\mathrm{L}$ en tres apiarios del municipio de Popayán. Facultad de ciencias Agropecuarias, 6 (2), 94-98.

Zavala, A., Colmo, I., Matalí, N., Piana, L., Olivier, B., Méndez, A., \& Vandame, R. (2013). Characterization of four typical honeys from highly diverse tropical ecosystems. Journal of Apicultural Research, 52(2): 24-34. 
PARA CITAR EL ARTÍCULO INDEXADO.

Guallpa Calva, M. Ángel, Espinoza Espinoza, A. E., Caranqui Aldaz, J. M., \& Guilcapi Pacheco, E. D. (2020). Potencial melífero de dos apiarios ubicados en los cantones Cevallos y Riobamba, Zona 3 interandina, Ecuador. ConcienciaDigital, 3(4.1), 46-61. https://doi.org/10.33262/concienciadigital.v3i4.1.1469

\section{Ciencia}

El artículo que se publica es de exclusiva responsabilidad de los autores y no necesariamente reflejan el pensamiento de la Revista Conciencia Digital.

El artículo queda en propiedad de la revista y, por tanto, su publicación parcial y/o total en otro medio tiene que ser autorizado por el director de la

Revista Conciencia Digital.

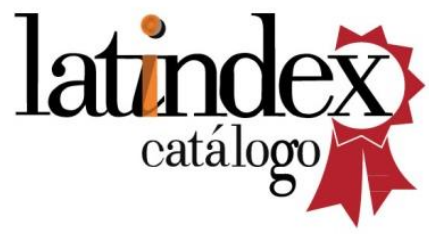

